\title{
Assessment and Formation Mechanism of Laser-Induced Periodic Surface Structures on Polymer Spin-Coated Films in Real and Reciprocal Space
}

\author{
Esther Rebollar, ${ }^{*+}$ Susana Pérez, ${ }^{\dagger}$ Jaime J. Hernández, ${ }^{\ddagger}$ Ignacio Martín-Fabiani, ${ }^{\ddagger}$ Daniel R. Rueda, \\ Tiberio A. Ezquerra, ${ }^{\ddagger}$ and Marta Castillejo ${ }^{+}$ \\ ${ }^{\dagger}$ Instituto de Química Física Rocasolano, Consejo Superior de Investigaciones Científicas, Serrano 119, 28006 Madrid, Spain \\ ${ }^{\ddagger}$ Instituto de Estructura de la Materia, Consejo Superior de Investigaciones Científicas, Serrano 121, 28006 Madrid, Spain
}

\begin{abstract}
In this work we evaluate the potential of grazing incidence X-ray scattering techniques in the investigation of laserinduced periodic surface structures (LIPSSs) in a series of strongly absorbing model spin-coated polymer films which are amorphous, such as poly(ethylene terephthalate), poly(trimethylene terephthalate), and poly (carbonate bisphenol A), and in a weaker absorbing polymer, such as semicrystalline poly(vinylidene fluoride), over a narrow range of fluences. Irradiation was performed with pulses of $6 \mathrm{~ns}$ at $266 \mathrm{~nm}$, and LIPSSs with period lengths similar to the laser wavelength and parallel to the laser polarization direction are formed by devitrification

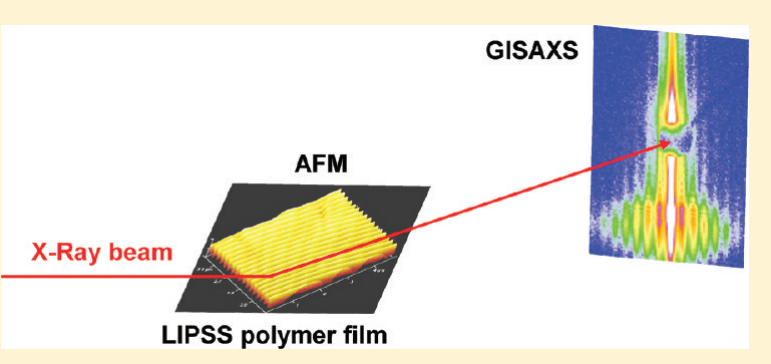
of the film surface at temperatures above the characteristic glass transition temperature of the polymers. No crystallization of the surface is induced by laser irradiation, and crystallinity of the material prevents LIPSS formation. The structural information obtained by both atomic force microscopy and grazing incidence small-angle X-ray scattering (GISAXS) correlates satisfactorily. Comparison of experimental and simulated GISAXS patterns suggests that LIPSSs can be well described considering a quasi-onedimensional paracrystalline lattice and that irradiation parameters have an influence on the order of such a lattice.
\end{abstract}

\section{INTRODUCTION}

Illumination of solid surfaces by intense laser pulses may provoke the appearance of spontaneous periodic surface structures with periodicities closely related to the wavelength of the irradiating laser. ${ }^{1}$ Formation of laser-induced periodic surface structures (LIPSSs) has been observed on the surface of metals, semiconductors, and dielectrics with lasers of different pulse durations from nanoseconds to femtoseconds and different wavelengths from UV to IR ${ }^{2-6}$ In the case of polymers, several studies have shown that irradiation by a polarized laser beam induces self-organized ripple structure formation within a narrow fluence range well below the ablation threshold. ${ }^{4-9}$ The period of the ripples $L$ depends on the laser wavelength and on the angle of incidence of the radiation and can be described by the following relation: ${ }^{10}$

$$
L=\frac{\lambda}{n-\sin (\theta)}
$$

where $\lambda$ is the laser wavelength, $n$ the effective refractive index of the material, and $\theta$ the angle of incidence of the laser beam. LIPSSs develop on the material surface as a result of treatment with a laser beam in such a way that the interference between the incoming and the surface-scattered waves causes an inhomogeneous intensity distribution, which together with a feedback mechanism results in the enhancement of the modulation depth. $^{7,11,12}$ However, the whole mechanism responsible for ripple formation is complex and still not well understood. In the case of polymers irradiated with nanosecond laser pulses, different processes, such as thermal and nonthermal scissoring of polymer chains, amorphization of crystalline domains, local surface melting, ablation, photolytic shrinkage, photooxidation, and material transport and rearrangement, have been proposed to be involved in ripple formation. ${ }^{4,7,8,11-14}$ The characteristic surface structures formed in this way can be used to tailor a great variety of surface properties, such as adhesion and friction, ${ }^{14-16}$ induced cell alignment, ${ }^{17}$ liquid crystal alignment, ${ }^{18,19}$ and colored images generated by superficial gratings. ${ }^{20}$

Typically, the more common way to investigate the morphology of LIPSSs is to carry out direct microscopic imaging of the surface by atomic force microscopy $(\mathrm{AFM})^{2,5-8,13,16,17,19}$ or alternatively by scanning electron microscopy (SEM). ${ }^{2-4,11,14,15,18}$ Quantitative information about the height and the period of the nanostructures is readily obtained by AFM over a range from several nanometers to several micrometers. However, AFM does not provide information on the layer underneath the structures. In fact, the main drawback of AFM is the lack of sensitivity to buried interfaces and the fact that images result in the

\footnotetext{
Received: February 3, 2011

Revised: $\quad$ March 8, 2011

Published: April 01, 2011
} 
convolution of the tip morphology with the real morphology of the relief. For large enough structures such as the ones investigated in this study, this latter point is a minor problem, but for structures with dimensions of a few nanometers, this could become a serious drawback. Contrary to AFM, X-ray scattering techniques do not provide a direct topography of the surface but information in reciprocal space. This information can be brought back to real space by means of mathematical processing of the $\mathrm{X}$-ray data. In principle, a periodic structure along a specific direction will be easily detected by X-ray diffraction techniques. The corresponding diffraction pattern provides statistical information integrated over a large sample area covered by the footprint of the incident beam on the surface of the material. Therefore, the scattering pattern can deliver structural information averaged over a macroscopic area of typically up to a few hundred square micrometers. Consequently, diffraction can be complementary to AFM for LIPSS characterization since it provides inspection of a much larger area than that observed by AFM. To accomplish X-ray diffraction experiments on surface nanostructures, it is very convenient to work under total X-ray reflection conditions. ${ }^{21-24}$ The technique of grazing incidence small-angle X-ray scattering (GISAXS) has been successfully applied to structurally characterize a great variety of soft matter thin films. ${ }^{21-24}$ Due to the fact that in GISAXS experiments diffraction is induced by a weak evanescent superficial wave, the use of X-ray synchrotron radiation is especially advantageous. ${ }^{24,25}$ In addition to structural changes involved in LIPSS formation, possible changes in the crystallinity of the polymer films can be studied by grazing incidence wide-angle X-ray scattering (GIWAXS). ${ }^{22,26}$

The main aim of this work is twofold: first, to evaluate the potential of grazing incidence X-ray scattering techniques in the investigation of LIPSSs and, second, to get further insight into

(a)

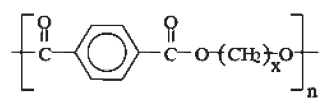

(b)

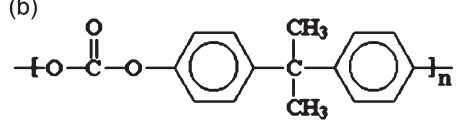

(c)

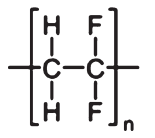

Figure 1. Chemical structures of the investigated polymers: (a) PET $(x=2)$, PTT $(x=3)$; (b) PC; (c) PVDF. the mechanisms that govern LIPSS formation in spin-coated polymer films. To accomplish these goals, LIPSSs have been induced by nanosecond UV pulses on different model polymers, including aromatic ones, such as poly(ethylene terephthalate), poly(trimethylene terephthalate), and poly(carbonate bisphenol A), or aliphatic ones, such as poly(vinylidene fluoride). These polymers present different properties, including the absorption coefficient at the different irradiation wavelengths, thermal transition temperatures, and crystallinity. The nanostructured surfaces were characterized in real space by AFM and in reciprocal space by GISAXS and GIWAXS. Our results suggest that the combination of techniques providing information in real and reciprocal space can constitute an advantageous strategy for the assessment of polymer LIPSS nanostructures.

\section{EXPERIMENTAL SECTION}

2.1. Materials. Four different polymers, poly(ethylene terephthalate) (PET) (Rhodia S80 from RhodiaSter), ${ }^{27}$ poly(trimethylene terephthalate) (PTT), ${ }^{28}$ poly(carbonate bisphenol A) (PC) (Lexan ML3021A, SABIC I-P (Innovative Plastics)), and poly(vinylidene fluoride) (PVDF) (Solef 6010, Solvay, Germany), were investigated in this work. Figure 1 shows their chemical structures, and some pertinent physical magnitudes are listed in Table 1. While PET, PTT, and PC can be easily obtained in the fully amorphous state at room temperature by fast quenching from the molten state, PVDF is typically semicrystalline. Previous to irradiation, UV-vis absorption spectra of the different polymers cast on quartz substrates were recorded with a UV-vis spectrophotometer (Perkin-Elmer U/V Lambda 16) in the range of $190-800 \mathrm{~nm}$. The linear absorption coefficients determined for the different wavelengths employed for irradiation are reported in Table 1. Polymer thin films were prepared by spin-coating on silicon wafers (100), polished on both surfaces. The wafers were previously cleaned with a piranha solution $\left(\mathrm{H}_{2} \mathrm{SO}_{4} / \mathrm{H}_{2} \mathrm{O}_{2}, 3: 1\right)$. PET and PTT were dissolved in trifluoroacetic acid (Sigma-Aldrich, reagent grade, $\geq 98 \%$ ), PC was dissolved in chloroform (Riedel-de Haën, 99\%), and PVDF was dissolved in N,N-dimethylacetamide (Sigma-Aldrich, 99\%) in the concentrations given in Table 2. A fixed amount of $0.1 \mathrm{~mL}$ of polymer solution was instantly dropped by a syringe on a rectangular ( 2 $\times 2.5 \mathrm{~cm}^{2}$ ) substrate cut out of a silicon wafer placed in the center of a rotating horizontal plate. The rotation speed, rotation time, and thickness of the polymer films, measured by AFM, are also listed in Table 2 . Besides semicrystalline samples of PVDF, and to assess how the crystallinity affects LIPSS formation, coated PTT samples were crystallized by thermal treatment (Mettler FP5, Mettler Instruments AG) at $255^{\circ} \mathrm{C}$ for $3 \mathrm{~min}$ and subsequently cooled to room temperature at $3{ }^{\circ} \mathrm{C} / \mathrm{min}$.

2.2. Laser Irradiation. Laser irradiation was carried out in ambient air, at normal incidence, with the linearly polarized laser beam of a Q-switched Nd:YAG laser (Quantel Brilliant B, pulse duration $\tau=6 \mathrm{~ns}$, full width at half-maximum) at a repetition rate of $10 \mathrm{~Hz}$. Fourth and fifth

Table 1. Weight Average Molecular Weight $\left(M_{\mathrm{w}}\right)$, Number Average Molecular Weight $\left(M_{\mathrm{n}}\right)$, Melting Temperature ${ }^{a}\left(T_{\mathrm{m}}\right)$, Glass Transition Temperature $\left(T_{\mathrm{g}}\right)$, Linear Absorption Coefficients $(\alpha)$ at 213 and $266 \mathrm{~nm}$, Density $(\rho)$, Specific Heat $(c)$, and Thermal Conductivity $(\kappa)$ of the Studied Polymer Materials

$\begin{array}{lccccccccc}\text { material } & M_{\mathrm{w}}(\mathrm{g} / \mathrm{mol}) & M_{\mathrm{n}}(\mathrm{g} / \mathrm{mol}) & T_{\mathrm{m}}\left({ }^{\circ} \mathrm{C}\right) & T_{\mathrm{g}}\left({ }^{\circ} \mathrm{C}\right) & \alpha_{213} \times 10^{3}\left(\mathrm{~cm}^{-1}\right) & \alpha_{266} & \rho^{42}\left(\mathrm{~kg} / \mathrm{m}^{3}\right) & c^{42}[\mathrm{~J} /(\mathrm{kg} \mathrm{K})] & \kappa^{42}[\mathrm{~W} /(\mathrm{m} \mathrm{K})] \\ \text { PET } & 27600 & 18800 & 252 & 75 & 38 & 18 & 1390 & 1172 & 0.15 \\ \text { PTT } & 42000 & 21000 & 229 & 44 & 52 & 26 & 1350 & 1359 \\ \text { PC } & 64900 & 30000 & 267 & 151 & 193 & 18 & 1200 & 1207 \\ \text { PVDF } & 288000 & 64000 & 248 & -35 & 0.6 & 0.4 & 1760 & 1100 & 0.22^{b}\end{array}$

${ }^{a}$ As estimated by calorimetry. ${ }^{b}$ Estimated as an intermediate value calculated from the values of $\kappa$ corresponding to PET and poly(butylene terephthalate) (PBT). ${ }^{42}$ 
Table 2. Experimental Conditions for Film Preparation by Spin-Coating, Thickness and Roughness $\left(R_{\mathrm{a}}\right)$ of the Coated Films, As Measured by AFM, and Fluence and Pulse Number Thresholds ( $F_{t h}$ and $N_{\text {th }}$, Respectively) for LIPSS Formation at the Indicated Conditions

\begin{tabular}{|c|c|c|c|c|c|c|c|}
\hline material & concn $(\mathrm{g} / \mathrm{L})$ & rotation speed (rpm) & time $(s)$ & thickness (nm) & $R_{\mathrm{a}}(\mathrm{nm})$ & $F_{\text {th }}\left(\mathrm{mJ} / \mathrm{cm}^{2}\right.$, at 1200 pulses $)$ & $N_{\text {th }}\left(\right.$ at $\left.6 \mathrm{~mJ} / \mathrm{cm}^{2}\right)$ \\
\hline PET & 20 & 2380 & 30 & $139 \pm 27$ & $0.6 \pm 0.1$ & 4 & 100 \\
\hline PTT & 20 & 2380 & 30 & $157 \pm 24$ & $1.1 \pm 0.5$ & 4 & 100 \\
\hline $\mathrm{PC}$ & 40 & 2380 & 30 & $130 \pm 21$ & $0.32 \pm 0.06$ & 5 & 900 \\
\hline PVDF & 10 & 1732 & 30 & $105 \pm 26$ & $21 \pm 17$ & & \\
\hline
\end{tabular}

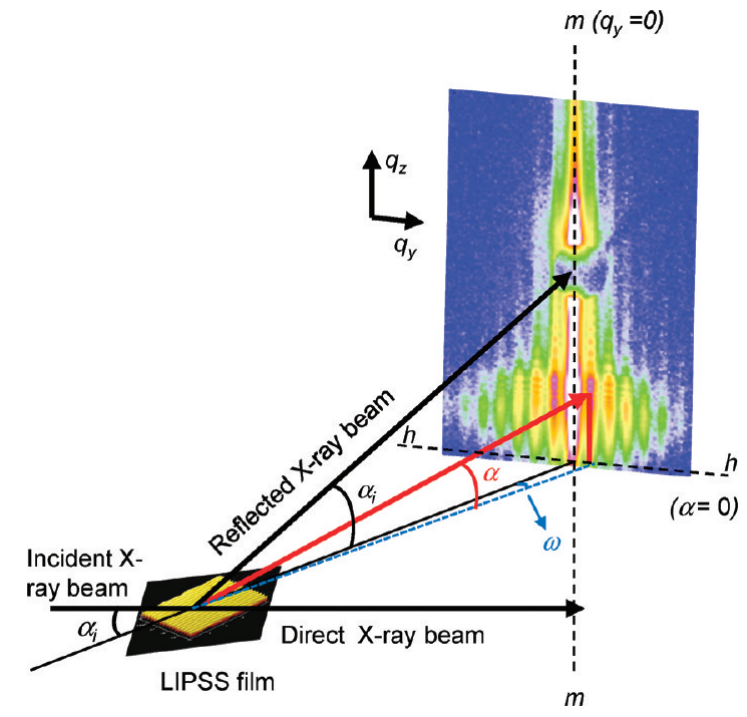

Figure 2. Schematic view of a GISAXS experiment. The scattering plane, containing both the direct and the specular beams, intersects the $2 \mathrm{D}$ detector along the meridian, $m-m$ line, of the GISAXS pattern. The horizon, $h-h$ line, is the intersection between the sample plane and the plane of the 2D detector, which are perpendicular to each other. Each point on the GISAXS pattern can be characterized by the exit angle, $\alpha$, and the out of scattering plane angle, $\omega$.

harmonics at 266 and $213 \mathrm{~nm}$, respectively, were used for the experiments. The fluences of irradiation were determined by measuring the laser energy in front of the sample with a joulemeter (Gentec-E, QE25SP-H-MB-D0) and calculating the area of the irradiated spots after delimitating the beam with an iris of $0.45 \mathrm{~cm}$ diameter. To produce LIPSSs, the spin-coated films of PET, PTT, PC, and PVDF were irradiated at normal incidence at $266 \mathrm{~nm}$, a wavelength where PET, PTT, and PC absorb efficiently (Table 1). The polymer films were irradiated with a constant number of pulses in the range of fluences of $1-15 \mathrm{~mJ} / \mathrm{cm}^{2}$ and as a function of the number of pulses at constant fluence. PVDF absorbs poorly at this wavelength, and irradiation was performed for fluences in the range of $1-140 \mathrm{~mJ} / \mathrm{cm}^{2}$. The effect of crystallinity was also studied by irradiating thermally treated PTT to $20 \mathrm{~mJ} / \mathrm{cm}^{2}$.

2.3. Surface Characterization and Analysis. The topography of the polymer films was examined via AFM (Nanoscope IIIA Multimode, Veeco) in tapping mode, and the images were analyzed with the software Nanoscope Analysis 1.10, obtaining the fast Fourier transform (FFT) of the AFM images. The original spin-coated samples present a flat surface, with mean roughness $\left(R_{\mathrm{a}}\right)$ values of $\leq 1 \mathrm{~nm}$. $R_{\mathrm{a}}$ values are listed in Table 2 and indicate the arithmetic average of the deviations in height from the center plane of the sample. Each $R_{\mathrm{a}}$ value corresponds to the average of three independent measurements in different locations of the film. The periods and heights of the LIPSSs were determined from
AFM analysis, averaging again the results of three different areas in each sample. Values of the period were derived from the FFT analysis (spatial frequency in the direction perpendicular to the LIPSS). Irradiated areas were also analyzed by GISAXS using the facilities of the BW4 beamline at Hamburger Synchrotronstrahlungslabor (HASYLAB) (Deutsches Elektronen-Synchrotron, DESY, Hamburg, Germany). A scheme of the experimental setup for GISAXS is shown in Figure 2. The information can be interpreted on the basis of the two orthogonal scattering vectors $q_{z}=(2 \pi / \lambda)\left(\sin \alpha_{i}+\sin \alpha\right)$ and $q_{y}=(2 \pi / \lambda) \sin \omega \cos \alpha$, which provide information about structural correlations perpendicular and parallel to the film plane, respectively. Lateral correlation between scattering objects on the film surface can induce some scattered intensity appearing out of the meridian (line $m-m$ in Figure 2). ${ }^{29}$ An X-ray wavelength of $\lambda=0.13808 \mathrm{~nm}$, with a beam size of $20 \times 40 \mu \mathrm{m}^{2}$, was used in our experiments. The scattered intensity was recorded by a Mar CCD detector of $2048 \times 2048$ pixels with a resolution of $79.1 \mu \mathrm{m}$ per pixel and a sample-to-detector distance of $2.211 \mathrm{~m}$. An incidence angle $\alpha_{i}=0.4^{\circ}$ was chosen, which is larger than that for polymer materials, and then full penetration in the sample is ensured. Samples were placed to guarantee that the beam was parallel to the direction of the LIPSS, and acquisition times between 40 and $600 \mathrm{~s}$ were used. The treatment of the GISAXS images was performed using the software FIT2D. ${ }^{30}$ GISAXS measurements provided an independent determination of the period, to be compared with the value obtained by AFM. On the other hand, GIWAXS measurements were also performed at the BW4 beamline at HASYLAB, and in this case the distance between the sample and the CCD detector was $0.17 \mathrm{~m}$. Analysis of 2D GIWAXS patterns can provide information on crystalline structure and crystal orientation among others.

\section{RESULTS}

3.1. LIPSSs in Amorphous Spin-Coated Polymers. Spincoated films of PET, PTT, and PC appear to be amorphous at room temperature as revealed by GIWAXS experiments. After laser irradiation at an appropriate fluence range, well below the ablation threshold, and with a given number of pulses, ripple formation is observed with a direction parallel to the polarization vector of the laser beam. As an example, Figure 3 displays typical AFM height images and the corresponding FFT images of areas of PTT irradiated with 1200 pulses at different fluences. For low fluences $\left(F<3 \mathrm{~mJ} / \mathrm{cm}^{2}\right)$, no morphological changes are induced on the polymer surface and roughness remains constant. At around $4 \mathrm{~mJ} / \mathrm{cm}^{2}$ (Figure $3 \mathrm{~b}$ ), surface modification starts, as revealed by an increase in roughness. For fluences above $4 \mathrm{~mJ} /$ $\mathrm{cm}^{2}$, structures start to be formed and develop in ripples at $F \geq 5$ $\mathrm{mJ} / \mathrm{cm}^{2}$. Ripples are parallel and well-defined in the range of 6-8 $\mathrm{mJ} / \mathrm{cm}^{2}$. For $F>8 \mathrm{~mJ} / \mathrm{cm}^{2}$, LIPSSs become distorted, and finally, above $14 \mathrm{~mJ} / \mathrm{cm}^{2}$, ablation of the polymer takes place, as displayed in Figure 3g. Similar results are observed for PET, while for PC the onset for ripple formation appears at a slightly higher fluence $\left(F \approx 5 \mathrm{~mJ} / \mathrm{cm}^{2}\right)$. 


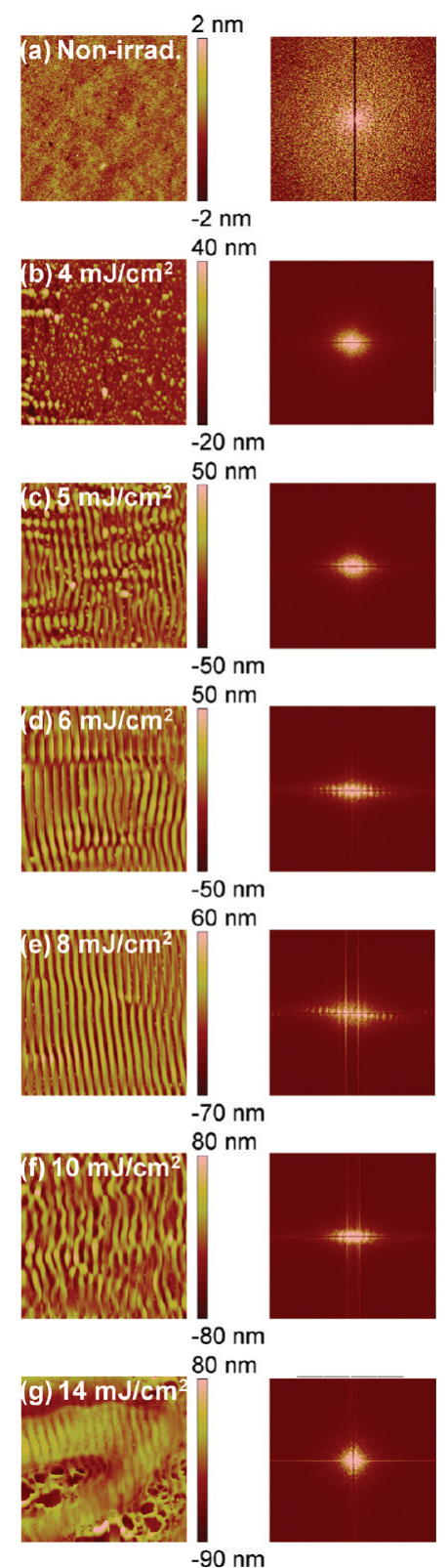

Figure 3. AFM (left) and corresponding FFT (right) images $(5 \times 5$ $\mu \mathrm{m}^{2}$ size) of PTT: nonirradiated (a); irradiated at $266 \mathrm{~nm}$ with 1200 pulses at fluences of (b) $4 \mathrm{~mJ} / \mathrm{cm}^{2}$, (c) $5 \mathrm{~mJ} / \mathrm{cm}^{2}$, (d) $6 \mathrm{~mJ} / \mathrm{cm}^{2}$, (e) 8 $\mathrm{mJ} / \mathrm{cm}^{2}$, (f) $10 \mathrm{~mJ} / \mathrm{cm}^{2}$, and $(\mathrm{g}) 14 \mathrm{~mJ} / \mathrm{cm}^{2}$.

For PET, PTT, and PC, the dependence of the period of LIPSSs on the fluence of irradiation at 1200 pulses, as derived from AFM analysis, is presented in Figure 4. As observed, the period increases in the fluence range of $4-6 \mathrm{~mJ} / \mathrm{cm}^{2}$ in the case of PET and PTT and remains practically constant up to $10 \mathrm{~mJ} /$ $\mathrm{cm}^{2}$, when the ripples start to disappear. In the case of PC, LIPSS formation starts at slightly higher fluences $\left(F \approx 5 \mathrm{~mJ} / \mathrm{cm}^{2}\right)$. For PTT an increase in the period from 205 to $250 \mathrm{~nm}$ is observed upon irradiation with 1200 pulses, while when the number of applied pulses increases to 6000 , the measured periods are $240-280 \mathrm{~nm}$, and no significant differences are observed in the range of fluences at which LIPSSs are formed. The periods obtained are similar to the laser wavelength employed, $266 \mathrm{~nm}$, as expected according to eq 1 . (a) PET

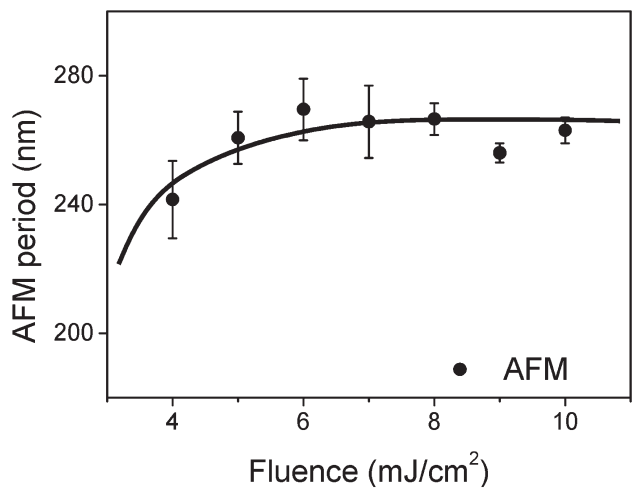

(b) PTT

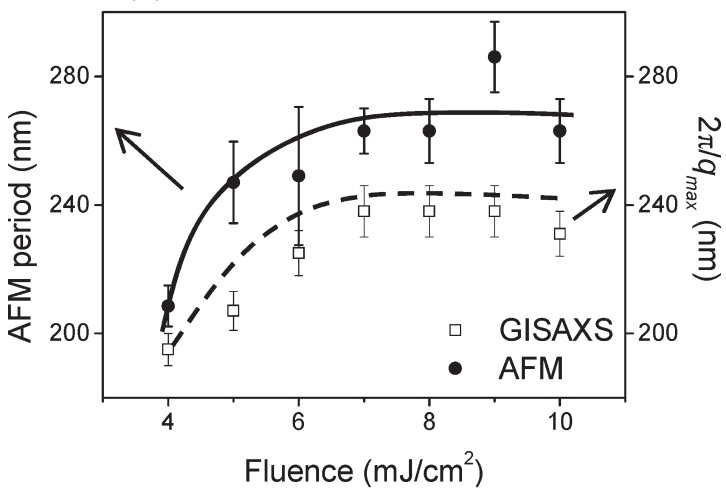

(c) PC

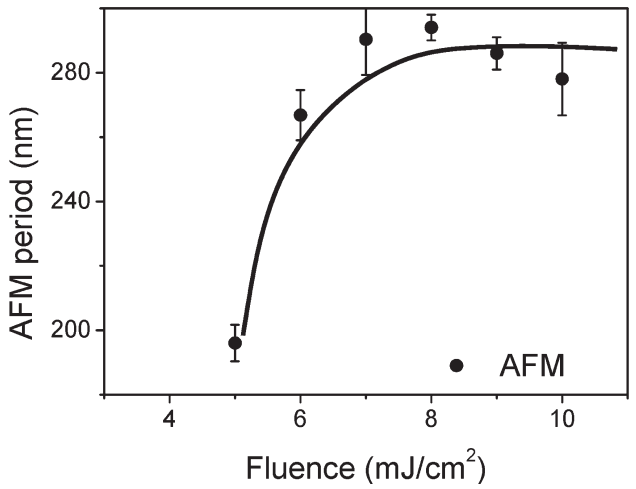

Figure 4. Period length as derived from AFM (left scale) of the nanostructures induced on (a) PET, (b) PTT, and (c) PC surfaces upon irradiation at $266 \mathrm{~nm}$ with 1200 pulses as a function of laser fluence. For PTT, the spacing of the first maximum from the GISAXS patterns (right scale) is also shown. The lines are plotted as visual guides.

Regarding the height of the ripples, as determined from the AFM images, there is not a clear dependence on the laser fluence of the values obtained. However, the height of the structures is systematically larger for the higher fluence in the range at which LIPSSs are observed, since the depth heated by laser irradiation increases with the fluence as discussed below. Typical values vary between 25 and $75 \mathrm{~nm}$ for PET, 40 and $100 \mathrm{~nm}$ for PTT, and 40 and $140 \mathrm{~nm}$ for PC.

The period of LIPSSs also exhibits a dependence on the number of pulses. Figure 5 shows AFM height images and their corresponding FFT images of LIPSSs created on the spin-coated PET films at $F=6 \mathrm{~mJ} / \mathrm{cm}^{2}$ with an increasing number of pulses. At this fluence LIPSSs are well-defined for a number of pulses $N$ 


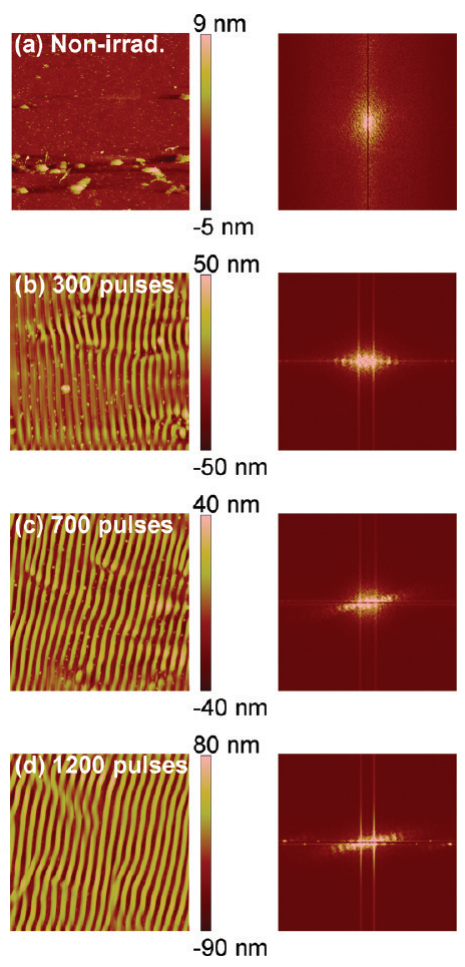

Figure 5. AFM images (left) and corresponding FFT images (right) of PET: nonirradiated (a) and irradiated at $266 \mathrm{~nm}$ and $6 \mathrm{~mJ} / \mathrm{cm}^{2}$ with (b) 300 pulses, (c) 700 pulses, and (d) 1200 pulses.

$>$ 100. Similar results are obtained for PTT. In the case of PC, 900 pulses are required to initiate LIPSS formation, as listed in Table 2. The dependence of the LIPSS period on the pulse number is exemplified in Figure 6 for PET, PTT, and PC. It is observed that the period of the structures increases with the number of pulses up to $N \approx 2000$ and then a plateau is reached. A qualitatively similar trend is found for PC, although shifted to a higher number of pulses. Regarding the heights of the nanostructures, no clear trend is observed, as in the case of the dependence on the fluence.

As far as GISAXS is concerned, Figure 7 shows, as an example, characteristic GISAXS patterns for the three aromatic polymers investigated in the range of laser parameters for LIPSS formation. In Figure 7 we have presented the AFM height images of the samples, the corresponding GISAXS patterns, and cuts of the scattered intensity as a function of $\omega$ extracted from the GISAXS patterns at $\alpha=0.2^{\circ}$. For the sake of comparison, results for a sample of PC below the LIPSS formation threshold $(N=6000$ pulses, $F=3 \mathrm{~mJ} / \mathrm{cm}^{2}$ ) are also shown (Figure $7 \mathrm{~d}$ ). Scattering maxima out of the meridian $(\omega \neq 0)$ are clearly visible in the range of LIPSS formation. This is illustrated in Figure 7 (right column) by the corresponding intensity profile across the horizontal direction (at a fixed $\alpha=0.2^{\circ}$ ). It is worth mentioning that the asymmetries observed in the GISAXS pattern are due to the small deviation in the parallelism of the structures (i.e., LIPSSs are not perfectly parallel along the whole inspected spot). Additionally, the background level in Figure $7 d$, which is higher than that obtained for the other samples, is presumably due to the contribution of the amorphous phase, which scatters at very low angles in this sample.

Spacing of the first maximum obtained from the GISAXS patterns can be determined through the expression $L=2 \pi / q_{y}^{\max }$,

\section{(a) PET}

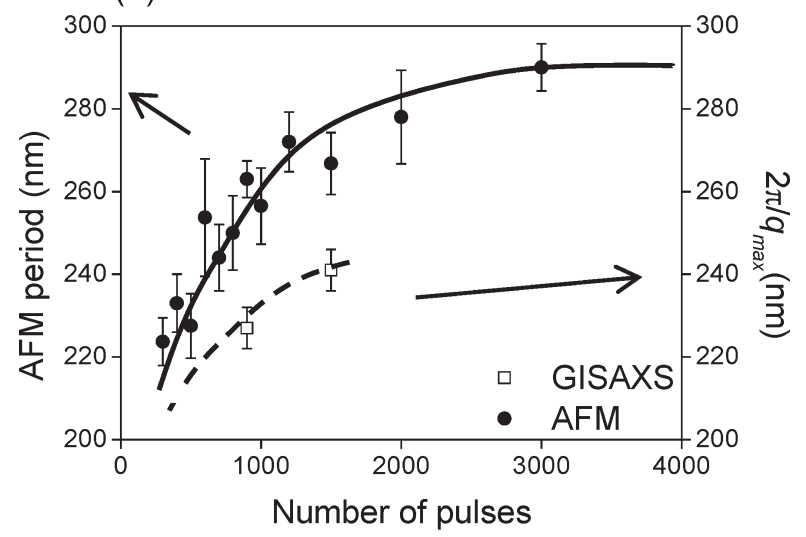

(b) PTT

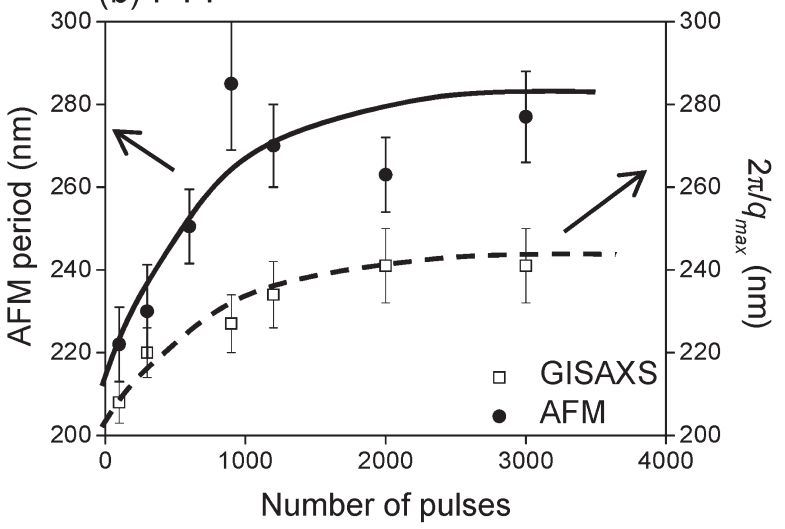

(c) PC

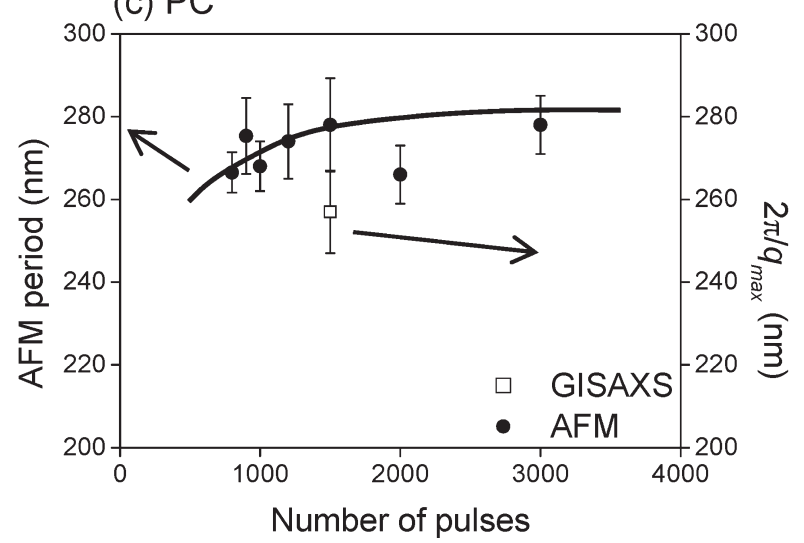

Figure 6. Period length as derived from AFM (left scale) and spacing of the first maximum obtained from the GISAXS patterns (right scale) as a function of the number of pulses on (a) PET surfaces upon irradiation at $266 \mathrm{~nm}$ and $6 \mathrm{~mJ} / \mathrm{cm}^{2}$, (b) PTT surfaces upon irradiation at $7 \mathrm{~mJ} / \mathrm{cm}^{2}$, and (c) PC surfaces upon irradiation at $6 \mathrm{~mJ} / \mathrm{cm}^{2}$. Lines are plotted to guide the eye.

where $q_{y}^{\max }$ is the $q$ value corresponding to the first intensity maximum (indicated by arrows in Figure 7). Spacing values derived from GISAXS for the irradiated polymers are shown in Figures $4 \mathrm{~b}$ and 6 . A very good qualitative correlation between AFM and GISAXS results is observed, although the values obtained by GISAXS are systematically lower than the ones obtained by AFM.

Additionally, periodic structures on PET, PTT, and PC were analyzed by GIWAXS to get information about eventual 
(a) PET, $6 \mathrm{~mJ} / \mathrm{cm}^{2}, 1500 \mathrm{p}$

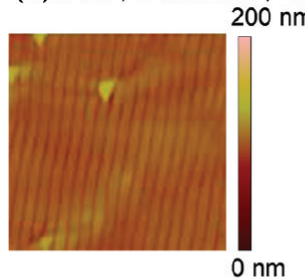

(b) PTT, $7 \mathrm{~mJ} / \mathrm{cm}^{2}, 300 \mathrm{p}$

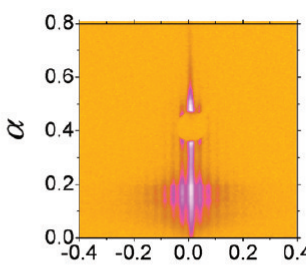

$\omega$

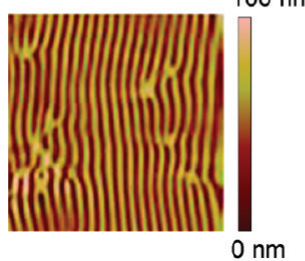

(c) $\mathrm{PC}, 7 \mathrm{~mJ} / \mathrm{cm}^{2}, 6000 \mathrm{p}$

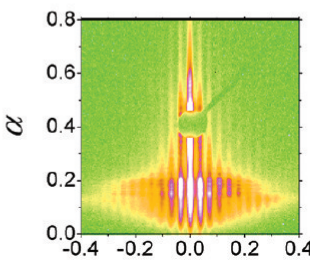

$\omega$
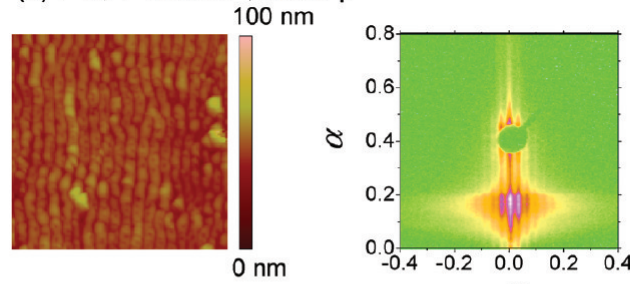

$\omega$

(d) PC, $3 \mathrm{~mJ} / \mathrm{cm}^{2}, 6000 \mathrm{p}$
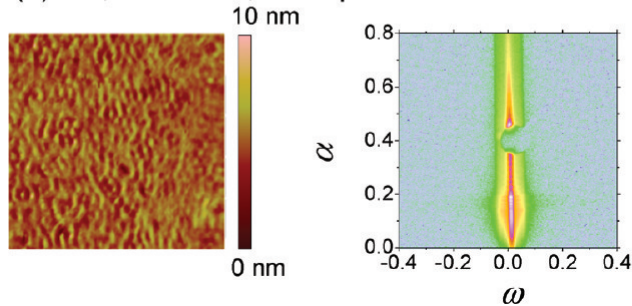
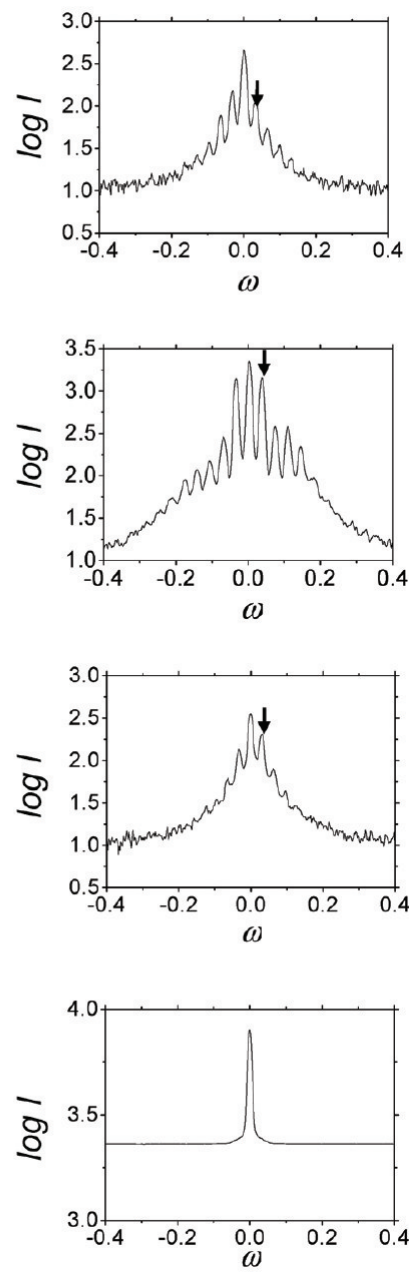

Figure 7. AFM images $\left(5 \times 5 \mu \mathrm{m}^{2}\right)$ (left), GISAXS patterns (center), and corresponding cuts at $\alpha=0.2^{\circ}$ (right) of PET irradiated with 1500 pulses at 6 $\mathrm{mJ} / \mathrm{cm}^{2}$ (a), PTT irradiated with 300 pulses at $7 \mathrm{~mJ} / \mathrm{cm}^{2}$ (b), and PC irradiated with 6000 pulses at $(\mathrm{c}) 7 \mathrm{~mJ} / \mathrm{cm}^{2}$ and $(\mathrm{d}) 3 \mathrm{~mJ} / \mathrm{cm}^{2} . \alpha$ and $\omega$ are given in degrees.

crystallization during LIPSS formation. Bragg reflections were not observed in any condition explored, indicating that the laserirradiated spin-coated polymer films remain in the amorphous state after LIPSS formation. These results confirm that no changes in crystallinity were induced in PET, PTT, and PC upon laser irradiation and structuring of the polymer surfaces.

3.2. LIPSSs in Semicrystalline Spin-Coated Polymers. While spin-coated PET, PTT, and PC films are amorphous at the present experimental conditions, PVDF is semicrystalline, and therefore, it presents the typical morphology of this kind of material, constituted by spherulites a few micrometers in size. Figure 8a shows an AFM picture of a PVDF sample before irradiation. Upon irradiation at $266 \mathrm{~nm}$ no LIPSSs were observed in a fluence range of $5-140 \mathrm{~mJ} / \mathrm{cm}^{2}$ (Figure $8 \mathrm{~b}, \mathrm{c}$ ). The same result was obtained when the sample was irradiated at $213 \mathrm{~nm}$ (not shown). However, for this material the linear absorption coefficient is very low at both 266 and $213 \mathrm{~nm}$ (Table 1) in comparison to that of the other polymers investigated. Then the lack of ripples could be related either to the initial polymer crystalline structure or to its weak absorption.

To elucidate the effect of crystallinity on LIPSS formation, amorphous PTT was thermally treated by heating to $255^{\circ} \mathrm{C}$, above its melting temperature, for $3 \mathrm{~min}$ and then cooled at $3{ }^{\circ} \mathrm{C} /$ min to obtain a semicrystalline film. The obtained surface morphology is shown in Figure 8d. Spherulites, typical of crystalline polymers, of ca. $100 \mu \mathrm{m}$ size are formed, and the average roughness is $2.9 \pm 0.4 \mathrm{~nm}$. The absorption coefficient of the material did not change after the thermal treatment, as confirmed by UV-vis absorption spectroscopy. Irradiation of the polymer film at $266 \mathrm{~nm}$, at the typical fluences which led to ripple formation in the case of amorphous PTT, did not result in the formation of LIPSSs (Figure 8e) even at fluences up to $100 \mathrm{~mJ} /$ $\mathrm{cm}^{2}$. However, above $150 \mathrm{~mJ} / \mathrm{cm}^{2}$ signs of polymer surface melting were evident, as shown in Figure $8 \mathrm{f}$, although the material does not reorganize itself into ordered structures.

\section{DISCUSSION}

4.1. Mechanism of LIPSS Formation in Spin-Coated Polymers. Regarding the mechanism of LIPSS formation, it is commonly accepted that interference between the incident laser beam and the surface-scattered wave plays an important role., ${ }^{70}$ The irradiated surface scatters the incident beam, which interferes with the surface wave, resulting in a modulated distribution 


\section{PVDF}
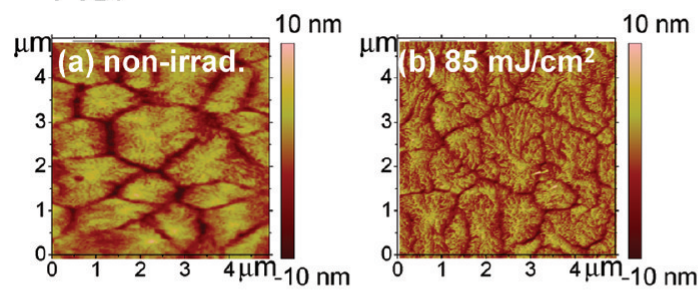

$10 \mathrm{~nm}$

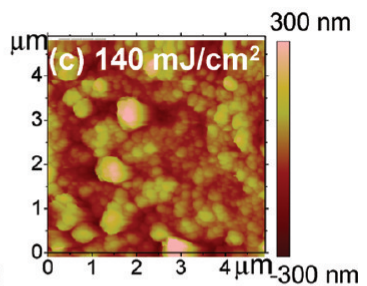

PTT thermally treated
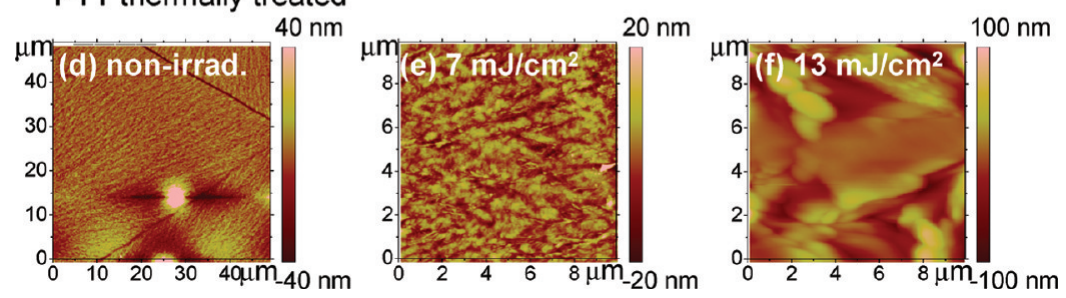

Figure 8. AFM images of PVDF [(a) nonirradiated, (b) irradiated at $266 \mathrm{~nm}$ with 6000 pulses at $85 \mathrm{~mJ} / \mathrm{cm}^{2}$, and (c) irradiated at $266 \mathrm{~nm}$ with 6000 pulses at $140 \mathrm{~mJ} / \mathrm{cm}^{2}$ ] and of crystallized PTT [(d) nonirradiated, (e) irradiated at $266 \mathrm{~nm}$ with 6000 pulses at $7 \mathrm{~mJ} / \mathrm{cm}^{2}$, and (f) irradiated at $266 \mathrm{~nm}$ with 6000 pulses at $13 \mathrm{~mJ} / \mathrm{cm}^{2}$.

of the energy on the surface. Some authors ${ }^{7,31}$ have proposed that the modulated energy disposal on the surface causes a similarly modulated heating, inducing temperature gradients and allowing diffusion of the polymeric chains. After repeated cycles of heating and cooling, accumulation of polymer motion leads to the formation of LIPSSs. ${ }^{31}$ In particular, for crystallizable materials, it has been suggested ${ }^{12,14}$ that heating may induce surface melting and subsequent cooling may cause crystallization at heated places. ${ }^{7}$ In this case, the initially smooth surface could increase its roughness through melting and crystallization cycles. Other possible explanations for LIPSS formation are related to periodic photolysis of the polymer chains or to periodic reaction of the excited surface with atmospheric oxygen, ${ }^{14}$ which lead to an increase of the UV absorbance and consequently to an increase of temperature. ${ }^{32}$

It is known that laser irradiation causes heating of the upper layer of the polymeric film. The corresponding temperature increase can be estimated as a function of time $t$ at different depths $x$ from the surface by solving the one-dimensional heat conduction equation ${ }^{7}$

$$
\frac{\partial^{2} T(x, t)}{\partial x^{2}}-a^{2} \frac{\partial T(x, t)}{\partial T}=-\frac{\alpha}{\kappa} P(t) \exp (-\alpha x) F_{0}
$$

$a^{2}=\rho c / k$, where $\rho$ is the density, $c$ the specific heat, and $\kappa$ the thermal conductivity of the material. $P(t)$ is a function that describes the temporal shape of the laser pulse; for the fourth harmonic of the Q-switched Nd:YAG laser used in the experiments herein, this function is adequately approximated by a modified Gaussian beam:

$$
P(t)=\frac{2 t}{\tau^{2}} \exp \left[-\left(\frac{t}{\tau}\right)^{2}\right]
$$

where the $t$ factor ensures that intensity vanishes at $t=0$ and $\tau$ is the pulse duration. This function is normalized to unity in the whole temporal range:

$$
\int_{0}^{\infty} P(t) \mathrm{d} t=\int_{0}^{\infty} \frac{2 t}{\tau^{2}} \exp \left[-\left(\frac{t}{\tau}\right)^{2}\right] \mathrm{d} t=1
$$

Formally, the heat conduction equation (eq 2) is solved as

$$
T(x, t)=T_{0}+\int \mathrm{d} t^{\prime} \int \mathrm{d} x^{\prime} G\left(x, t ; x^{\prime}, t^{\prime}\right) J\left(x^{\prime}, t^{\prime}\right)
$$

where $T_{0}$ is the initial temperature, $J\left(x^{\prime}, t^{\prime}\right)$ is defined as

$$
J\left(x^{\prime}, t^{\prime}\right)=t \exp \left(-t^{2}\right) \exp \left[-\left(\frac{x \alpha \sqrt{\tau}}{a}\right)\right]
$$

and $G\left(x, t ; x^{\prime}, t^{\prime}\right)$ is Green's function of the heat equation operator $O$

$$
O=\frac{\partial}{\partial t}-\frac{\partial^{2}}{\partial x^{2}}
$$

satisfying

$$
O G\left(x, t ; x^{\prime}, t^{\prime}\right)=\partial\left(x-x^{\prime}\right) \partial\left(t-t^{\prime}\right)
$$

and known from quantum mechanics as

$$
G\left(x, t ; x^{\prime}, t^{\prime}\right)=\frac{1}{\sqrt{4 \pi\left(t-t^{\prime}\right)}} \exp \left[-\frac{\left(x-x^{\prime}\right)^{2}}{4\left(t-t^{\prime}\right)}\right]
$$

Finally, the temperature is given by

$$
\begin{aligned}
T(x, t)= & T_{0}+\int_{0}^{t} \mathrm{~d} t^{\prime} \int_{-\infty}^{\infty} \mathrm{d} x^{\prime} \frac{t^{\prime}}{\sqrt{4 \pi\left(t-t^{\prime}\right)}} \exp \left[-\frac{\left(x-x^{\prime}\right)^{2}}{4\left(t-t^{\prime}\right)}\right] \\
& \exp \left(-t^{\prime 2}\right) \exp \left[\left(\frac{-\alpha \sqrt{\pi}}{a}\right)\right]\left|x^{\prime}\right|
\end{aligned}
$$

This function is a solution of eq 2, which can be verified by applying partial derivatives with the appropriate boundary conditions. Finally, the temperature is obtained by calculating numerically the integral of eq 10 and assuming an initial temperature of $23{ }^{\circ} \mathrm{C}$. The integration is also carried out for negative $x$ values, but since the absolute value of the integrand is taken, it is guaranteed that the derivative at $x=0$ vanishes. For the sake of simplification, the temperature dependence of the polymer physical parameters were not considered in the calculation, 
(a) PET

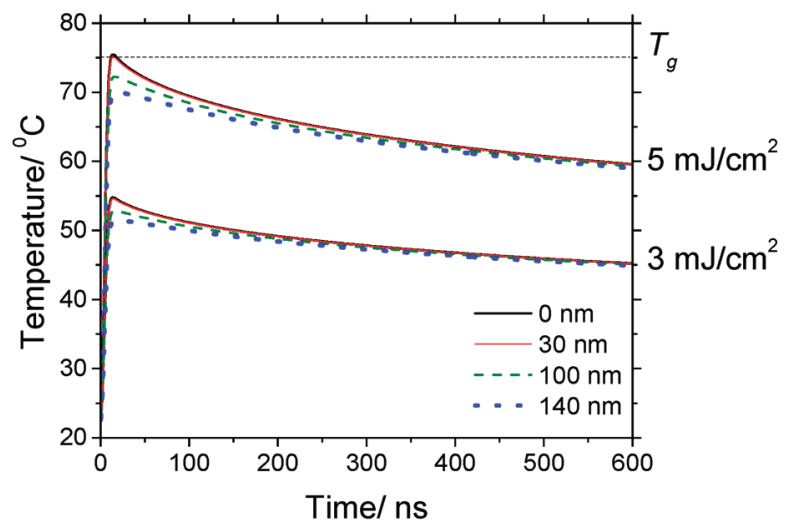

(b) PC

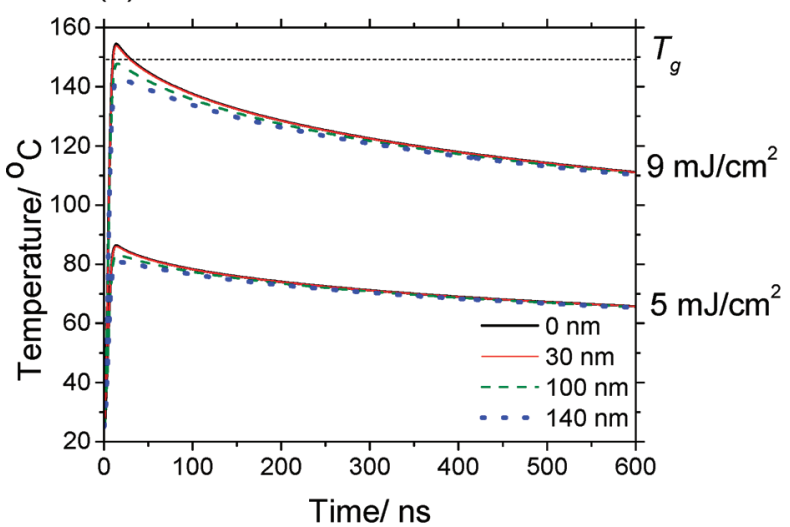

(c) PVDF

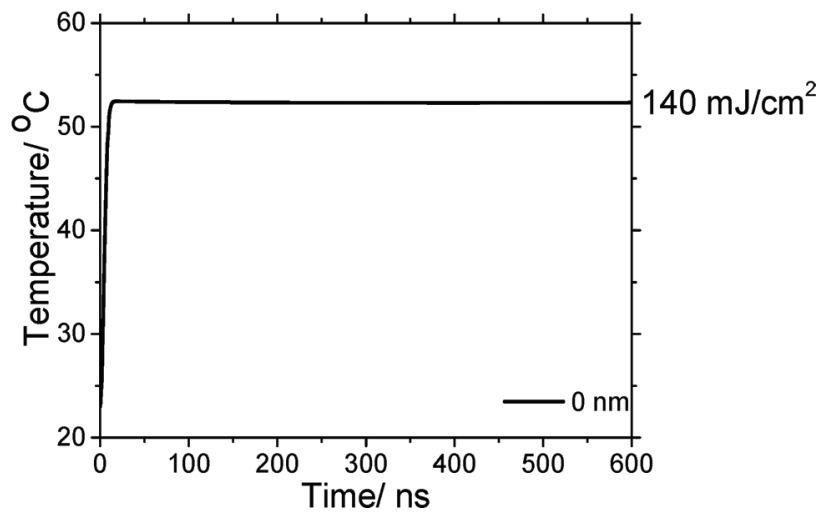

Figure 9. Time dependence of the temperature reached at different depths for $266 \mathrm{~nm}$ irradiation at the indicated fluences for (a) PET (temperature curves at the surface and at a depth of $30 \mathrm{~nm}$ overlap), (b) PC (temperature curves at the surface and at a depth of $30 \mathrm{~nm}$ overlap), and (c) PVDF.

together with the possible changes in the thermal properties of the surface during the thermal and chemical transformation caused by the previous laser pulses (i.e., incubation effects). These effects are estimated to have a minimum influence on the final temperature values. The magnitudes of the properties of the different polymers used for calculating the temperature profiles are given in Table 1.

The results of the calculations for PET, PC, and PVDF are illustrated in Figure 9. It is shown that for PET irradiated below 4
$\mathrm{mJ} / \mathrm{cm}^{2}$, the highest attained temperature is $65^{\circ} \mathrm{C}$, which is below the glass transition point $\left(T_{\mathrm{g}}\right)$ of PET. However, when the fluence reaches $5 \mathrm{~mJ} / \mathrm{cm}^{2}$, a layer of around $30 \mathrm{~nm}$ heats to a temperature above $T_{\mathrm{g}}$. These results suggest that, to obtain LIPSSs in these polymers, a minimum fluence value is necessary to ensure that the surface temperature overcomes the $T_{\mathrm{g}}$ values. In our case, for fluences above $5 \mathrm{~mJ} / \mathrm{cm}^{2}$, the surface temperature is expected to surpass $T_{\mathrm{g}}$ (Table 1 ), inducing surface devitrification, a phenomenon understood as the transformation from a glassy to a soft state, and therefore allowing polymer segmental and chain dynamics. This value of fluence is slightly higher than that corresponding to the experimental threshold for ripple formation for PET and PTT $\left(4 \mathrm{~mJ} / \mathrm{cm}^{2}\right)$, but it should be remembered that a simplified temperature calculation was performed. The temperature increase above $T_{\mathrm{g}}$ is expected to induce an increase in surface roughness caused by capillary waves, ${ }^{33}$ enhancing surface inhomogeneities and facilitating the feedback mechanism involved in LIPSS formation. For PC, as mentioned above, higher fluences and numbers of pulses are needed to induce LIPSSs, as expected due to its higher $T_{\mathrm{g}}$ (Table 1). In fact, some authors have proposed that a higher $T_{\mathrm{g}}$ tends to prolong the "incubation" period needed for LIPSS formation. ${ }^{31}$ As shown in Figure $9 \mathrm{~b}$, at the fluence of $5 \mathrm{~mJ} / \mathrm{cm}^{2}$ the temperature increase is not enough to reach the polymer $T_{\mathrm{g}}$, but at a fluence of $9 \mathrm{~mJ} /$ $\mathrm{cm}^{2}$, at which LIPSSs are optimally formed, a layer with a thickness of around $100 \mathrm{~nm}$ is heated above $T_{\mathrm{g}}$.

At a fluence of $10 \mathrm{~mJ} / \mathrm{cm}^{2}$, which corresponds to the value at which LIPSSs become distorted in PET, the temperature attained on the surface doubles the value of $T_{g}$, and the whole film is affected. In this case devitrification of the complete film develops. Under these circumstances film dewetting is likely to occur. As is evident in Figure $3 g$, at higher fluences ablation of the film material takes place, as should be expected due to the high temperatures attained, close to $200{ }^{\circ} \mathrm{C}$, at a fluence of $14 \mathrm{~mJ} / \mathrm{cm}^{2}$.

The estimation of the temperature increase also allows discussion of the increase of the ripple period as a function of the fluence in the restricted range at which ripples are formed $\left(F=4-10 \mathrm{~mJ} / \mathrm{cm}^{2}\right)$. In fact, the increase of fluence leads to an increase of the superficial temperature, which in turn generates a "softer material" with lower superficial viscosity. ${ }^{34}$ This allows the formation of wider structures characterized by larger periods at higher fluences.

The requirement of reaching temperatures above $T_{\mathrm{g}}$ for LIPSS formation implies that ripples are not expected for weakly absorbing amorphous polymers where the laser light penetration depth is higher and the temperatures attained at the surface are not high enough to soften the surface. One interesting aspect that needs to be discussed concerns the absence of crystallization effects, as revealed by GIWAXS, on both PET and PTT. It is known that both polymers are crystallizable above $T_{\mathrm{g}}$ with crystallization kinetics providing mean crystallization times in the range of minutes or even seconds. ${ }^{35}$ It is worth mentioning that, as shown in Figure 9a, although the increase in temperature induced by a single $6 \mathrm{~ns}$ pulse may reach values above $T_{\mathrm{g}}$, there is a subsequent cooling process which returns the temperature surface to its initial value after a few microseconds in the case of PET, PTT, and PC. This time interval can be determined by calculating the characteristic thermal diffusion time, ${ }^{36} t_{\mathrm{d}}$, which is given by $t_{\mathrm{d}}=1 / \gamma \alpha^{2}$, where $\gamma$ is the thermal diffusivity, $\gamma=\kappa / \rho c$, and $\alpha$ is the absorption coefficient (Table 1 ). The calculated thermal diffusion time is $t_{\mathrm{d}} \approx 3 \mu$ s for PET, $t_{\mathrm{d}} \approx 1 \mu \mathrm{s}$ for PTT, and $t_{\mathrm{d}} \approx 2 \mu$ s for PC. Experimentally, a subsequent pulse, which reaches the surface $100 \mathrm{~ms}$ after the previous one, finds the 
surface with a recovered initial temperature. Consequently, cumulative thermal effects due to repetitive irradiation do not take place, and the time interval in which the polymer surface is above $T_{\mathrm{g}}$ is on the order of nanoseconds. Within this short time interval, polymer diffusion is not important enough to reach the crystal nucleation step.

In the particular case of semicrystalline polymers, such as crystallized PTT or PVDF, the thermal properties are governed not only by the glass transition temperature but also by the melting temperature $\left(T_{\mathrm{m}}\right)$. The absence of LIPSS formation for semicrystalline PTT, in the fluence range where LIPSSs appear for the amorphous specimens, can be explained by considering that significant polymer dynamics is not reached below the melting point, which is much higher than $T_{\mathrm{g}}$, since the crystalline phase constitutes a robust scaffold providing mechanical stability to the material. For PVDF, which is both crystalline and weakly absorbing at the laser wavelength of $266 \mathrm{~nm}$, the time dependence of the surface temperature is shown in Figure $9 b$ for irradiation at $140 \mathrm{~mJ} / \mathrm{cm}^{2}$. The temperature calculated for all depths along the whole film thickness is constant with a value of around $50^{\circ} \mathrm{C}$. This temperature is well below the melting point of this material $\left(T_{\mathrm{m}}=248{ }^{\circ} \mathrm{C}\right.$, Table 1$)$, which may explain the absence of induced modifications in the range of fluences investigated. Due to the low absorbance, the temperature profile for PVDF presents some remarkable differences with respect to those of PET and PC. First, the temperature is significantly lower at comparable fluences, and second, the thermal relaxation process is much slower, with $t_{\mathrm{d}} \approx 11 \mathrm{~ms}$ being of the same order as the interval between successive pulses under the $10 \mathrm{~Hz}$ repetition rate used in the experiments. Accordingly, a cumulative temperature increase is expected with the accumulation of pulses and explains the film melting observed in the AFM image of Figure 8c.

It is important to mention that the thermal dissipation effect of the silicon substrate on the temperature increase has not been taken into account. From the values of the linear absorption coefficients of PET, PTT, and PC listed in Table 1, the irradiation depth can be calculated to be a few hundred nanometers. This implies that a negligible amount of laser radiation reaches the silicon substrate, and therefore, the contribution of silicon in the spatiotemporal temperature evolution can be disregarded. Additionally, according to the thermal properties of silicon and, in particular, its thermal conductivity $(149 \mathrm{~W} /(\mathrm{m} \mathrm{K}))$, the substrate would induce the cooling of the closest polymer layers. This effect would moderate the heating to the outer polymer layers, which in turn would favor LIPSS formation. The cooling effect of the substrate could be relevant in the case of weakly absorbing polymers as is the case for PVDF, since the calculated optical penetration depth is as high as $25 \mu \mathrm{m}$. However, the experimental results show that PVDF is melted upon irradiation with 6000 pulses at $140 \mathrm{~mJ} / \mathrm{cm}^{2}$ (Figure $8 \mathrm{c}$ ), indicating that even if the cooling effect due to the substrate is present, a cumulative temperature increase takes place and governs the process.

As noted above, the estimation of the film surface temperatures allows the discussion of the main thermal processes involved in LIPSS formation. In previous works by other authors, melting and even recrystallization of the heated areas have been proposed to be relevant for LIPSS formation. ${ }^{7}$ However, as shown here, the temperatures calculated together with the GIWAXS results suggest disregarding the role of melting and crystallization during LIPSS formation in the investigated case and rather LIPSS formation in amorphous spin-coated polymer
Table 3. Geometric Parameters of the Box Used for GISAXS Simulations: Width $(2 R)$, Height $(H)$, Period $(L)$, and Disorder Factor $(\omega)^{a}$

\begin{tabular}{ccccc} 
material & $2 R(\mathrm{~nm})$ & $H(\mathrm{~nm})$ & $L(\mathrm{~nm})$ & $\omega(\mathrm{nm})$ \\
PET & 180 & 19 & 263 & 78 \\
PTT & 120 & 20 & 220 & 47 \\
PC & 176 & 17 & 278 & 75 \\
${ }^{a}$ A constant box length $(W=1000 \mathrm{~nm})$ & was considered. \\
\hline
\end{tabular}

films takes place by devitrification of the film surface at temperatures above their characteristic $T_{\mathrm{g}}$. Moreover, for semicrystalline PVDF, irradiation at high fluences leads to melting of the film, but rearrangement of the material into periodic structures does not occur.

4.2. Assessment of LIPSSs by GISAXS. As far as the comparison between AFM and GISAXS data is concerned, a good agreement, within $\sim 20 \%$, is observed between the period values. The fact that the values of the period obtained by GISAXS are systematically smaller than those obtained by AFM analysis can be understood considering the nature of the GISAXS signal. Under the premises of the effective surface approximation, ${ }^{39}$ the scattered intensity $I(q)$ is approximately proportional to the form and the structure factors $P(q)$ and $S(q)$, respectively, by $I(q) \propto$ $P(q) S(q)$. The form factor $P(q)$ is related to the shape of the scattering object through the Fourier transform of its electron density distribution. In turn, the structure function $S(q)$ is related to the spatial correlation among the scattering objects and therefore contains information about the nanostructure through the pair correlation function. ${ }^{37}$ In general, a precise analysis of GISAXS patterns requires more sophisticated tools than the mere application of the Bragg law $\left(L=2 \pi / q_{\max }\right)$ to the observed intensity maxima to extract the underlying structural features of the investigated sample. ${ }^{38}$ To get better insight into the type of order of the LIPSS structures obtained on the spin-coated polymer films, a simulation of the GISAXS patterns was performed with the software package IsGISAXS. ${ }^{39,40}$ The distorted wave Born approximation (DWBA) was used, assuming a simple nanostructure consisting of polymer boxes standing on the polymer film. The dimensions of the boxes used to model the nanostructures are those determined by AFM, i.e., width $(2 R)$, height $(H)$, and period $(L)$, listed in Table 3, with a length $(W)$ of $1000 \mathrm{~nm}$ (see Figure 10a). Gaussian distributions for $R$ and $H$ have been assumed, and their values have been directly taken from the AFM determination. Disorder is introduced via a onedimensional paracrystal approach, in which the long-range order is lost gradually in a probabilistic way. ${ }^{37,39-41}$ The probability of finding a particle at a distance $L$ is defined by a function $p(x)$ that is considered as a Gaussian in the present simulation:

$$
p(x)=\frac{1}{\omega \sqrt{2 \pi}} \exp \left[-\frac{(x-L)^{2}}{2 \omega^{2}}\right]
$$

As the $\omega / L$ ratio increases, structure disorder increases, while for small $\omega / L$ values a $1 D$ crystalline lattice is obtained. Figure 10 shows the GISAXS patterns of the PET, PTT, and PC samples shown in Figure 7. For the sake of comparison the intensity values have been normalized to the corresponding value at $\omega=$ $0^{\circ}$. The IsGISAXS-simulated patterns have also been included in Figure 10. Table 3 summarizes the parameters used for the 
(a) LIPSS geometric parameters

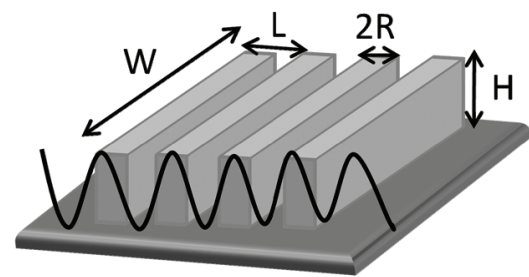

(b) PET, $6 \mathrm{~mJ} / \mathrm{cm}^{2}, 1500 \mathrm{p}$

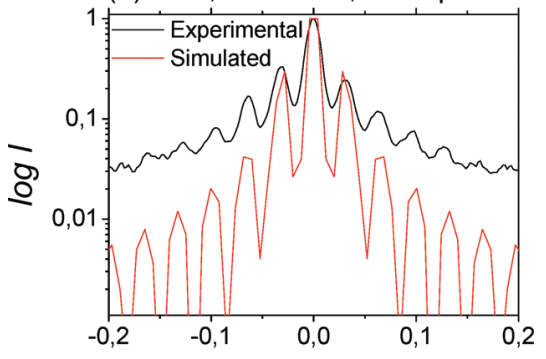

(c) PTT, $7 \mathrm{~mJ} / \mathrm{cm}^{2}, 300 \mathrm{p}$

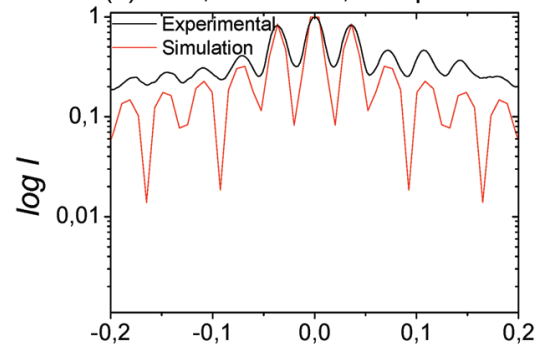

(d) PC, $7 \mathrm{~mJ} / \mathrm{cm}^{2}, 6000 \mathrm{p}$

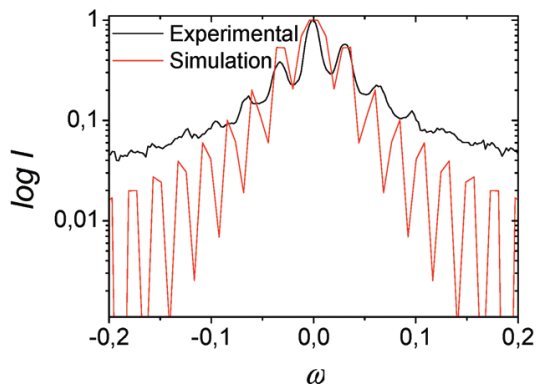

Figure 10. LIPSS film geometric parameters used for GISAXS simulation and profile of an experimental relief (a). Comparison of experimental and simulated cuts of GISAXS patterns at $\alpha=0.2^{\circ}$ for (b) PET irradiated with 1500 pulses at $6 \mathrm{~mJ} / \mathrm{cm}^{2}$, (c) PTT irradiated with 300 pulses at $7 \mathrm{~mJ} / \mathrm{cm}^{2}$, and (d) PC irradiated with 6000 pulses at $7 \mathrm{~mJ} / \mathrm{cm}^{2}$.

simulation and the value of the disorder factor $\omega$ which better fits the GISAXS patterns. These simulations strongly suggest that LIPSSs can be well described considering a quasi-one-dimensional paracrystalline lattice and that the irradiation parameters (i.e., fluence and number of pulses) have an influence on the order of such a lattice. As the simulation yields the order factor of the structures, it is possible for each material and laser wavelength to derive the optimal fluence and number of pulses that optimize LIPSS formation. Furthermore, considering that the structural parameters for the fittings are taken directly from those estimated by AFM, it is clear that both techniques are consistent with each other. Further work to systematically relate the degree of structural order with LIPSS conditions is in progress.

\section{CONCLUSIONS}

Laser-induced periodic surface structures with periods similar to the laser wavelength and parallel to the laser polarization direction are observed in amorphous spin-coated films of strongly absorbing model amorphous polymers, such as PET, PTT, and PC, in a narrow range of fluences. No LIPSSs are formed in the investigated spin-coated semicrystalline films of PVDF and semicrystalline films of PTT. Calculation of the temperature increase induced upon laser irradiation indicates that LIPSS formation in amorphous spin-coated polymer films takes place by devitrification of the film surface at temperatures above the characteristic $T_{\mathrm{g}}$ of the polymers. However, no crystallization is induced by the laser irradiation. Grazing incidence X-ray scattering techniques (GISAXS and GIWAXS) are introduced for the study of LIPSSs to mainly assess morphology order over large sample areas. These results validate GISAXS as an appropriate technique for the analysis of this kind of nanostructure and pave the way for further studies involving the online monitoring of LIPSSs by X-ray synchrotron techniques.

\section{AUTHOR INFORMATION}

\section{Corresponding Author}

*E-mail: e.rebollar@iqfr.csic.es.

\section{ACKNOWLEDGMENT}

Funding from the Ministerio de Educación y Ciencia, Spain (Projects CTQ2007-60177, CTQ2010-15680, PMAT200906991-E, and MAT2009-07789), is gratefully acknowledged. The experiments performed at BW4 in HASYLAB (DESY, Germany) were supported by the European Community (Contract RII3-CT-2004-506008 (Integrating Activity on Synchrotron and Free Electron Laser Science)). We thank J. Perlich for his support during the measurements at HASYLAB. E.R. thanks the Ministerio de Ciencia e Innovación (MICINN), Spain, for a Juan de la Cierva contract, and S.P., J.J.H, and I. M.-F. acknowledge MICINN for Formación de Personal Investigador fellowships. Assistance from A. González-Arroyo with temperature calculations and $\mathrm{M}$. Oujja with the experimental setup is also acknowledged. We thank Z. Roslaniec and A. Szymczyk for giving us the PTT sample.

\section{REFERENCES}

(1) Guosheng, Z.; Fauchet, P. M.; Siegman, A. G. Phys. Rev. B 1982, 26, 5366.

(2) Reif, J.; Varlamova, O.; Ratzke, M.; Schade, M.; Leipner, H. S.; Arguirov, T. Appl. Phys. A: Mater. Sci. Process. 2010, 101, 361.

(3) Bonse, J.; Krüger, J. J. Appl. Phys. 2010, 108, 034903.

(4) Bolle, M.; Lazare, S.; Le Blanc, M.; Wilmes, A. Appl. Phys. Lett. 1992, 60, 674 .

(5) Csete, M.; Hild, S.; Plett, A.; Zlemann, P.; Bor, Zs.; Marti, O. Thin Solid Films 2004, 453, 114.

(6) Li, M.; Lu, Q. H.; Yin, J.; Sui, Y.; Li, G.; Qian, Y.; Wang, Z. G. Appl. Surf. Sci. 2002, 193, 46.

(7) Csete, M.; Bor, Z. Appl. Surf. Sci. 1998, 133, 5.

(8) Csete, M.; Eberle, R.; Pietralla, M.; Marti, O.; Bor, Z. Appl. Surf. Sci. 2003, 208-209, 474.

(9) Lazare, S.; Benet, P. J. Appl. Phys. 1993, 74, 4953.

(10) Bäuerle, D. Laser Processing and Chemistry; Springer-Verlag: Berlin, 2000.

(11) Bolle, M.; Lazare, S. J. Appl. Phys. 1993, 73, 3516. 
(12) Prendergast, Ú.; Kudzma, S.; Sherlock, R.; O’Connell, C.; Glynn, T. Proc. SPIE-Int. Soc. Opt. Eng. 2007, 6458, 64581V.

(13) Csete, M.; Marti, O.; Bor, Zs. Appl. Phys. A: Mater. Sci. Process. 2001, 73, 521 .

(14) Bolle, M.; Lazare, S. Appl. Surf. Sci. 1993, 65-66, 349.

(15) Bolle, M.; Lazare, S. Appl. Surf. Sci. 1993, 69, 31.

(16) Slepička, P.; Rebollar, E.; Heitz, J.; Švorčík, V. Appl. Surf. Sci. 2008, 254, 3585.

(17) Rebollar, E.; Frischauf, I.; Olbrich, M.; Peterbauer, T.; Hering, S.; Preiner, J.; Hinterdorfer, P.; Romanin, C.; Heitz, J. Biomaterials 2008, 29, 1796.

(18) Niino, H.; Kawabata, Y.; Yabe, A. Jpn. J. Appl. Phys. 1989, 28, L2225.

(19) Lu, H. W.; Lu, Q. H.; Chen, W. T.; Xu, H. J.; Yin, J. Mater. Lett. 2004, 58, 29.

(20) Lochbihler, H. Opt. Express 2009, 12189, 17.

(21) Hernández, J. J.; Rueda, D. R.; García-Gutiérrez, M. C.; Nogales, A.; Ezquerra, T. A.; Soccio, M.; Lotti, N.; Munari, A. Langmuir 2010, 26, 10731.

(22) Jin, S.; Hirai, T.; Ahn, B.; Rho, Y.; Kim, K.-W.; Kakimoto, M.-A.; Gopalan, P.; Hayakawa, T.; Ree, M. J. Phys. Chem. B 2010, 114, 8033.

(23) Sohn, K. E.; Kojio, K.; Berry, B. C.; Karim, A.; Coffin, R. C.; Bazan, G. C.; Kramer, E. J.; Sprung, M.; Wang, J. Macromolecules 2010, 43, 3406.

(24) Müller-Buschbaum, P. Anal. Bioanal. Chem. 2003, 376, 3.

(25) Naudon, A.; Babonneau, D.; Thiaudiere, D.; Lequien, S. Physica B 2000, 283, 69.

(26) Perlich, J.; Rubeck, J.; Botta, S.; Gehrke, R.; Roth, S. V.; Ruderer, M. A.; Prams, S. M.; Rawolle, M.; Zhong, Q.; Körstgens, V.; Müller-Buschbaum, P. Rev. Sci. Instrum. 2010, 81, 105105.

(27) Alvarez, C.; Sics, I.; Nogales, A.; Denchev, Z.; Funari, S. S.; Ezquerra, T. A. Polymer 2004, 45, 3953.

(28) Szymczyk, A. Eur. Polym. J. 2009, 45, 2653.

(29) Rueda, D. R.; Nogales, A.; Hernández, J. J.; García-Gutiérrez, M. C.; Ezquerra, T. A.; Roth, S. V.; Zolotukhin, M. G.; Serna, R. Langmuir 2007, 23, 12677.

(30) The FIT2D Home Page. http://www.esrf.eu/computing/ scientific/FIT2D/ (accessed March 15, 2010).

(31) Li, M.; Lu, Q. H.; Yin, J.; Qian, Y.; Wang, Z. G. Mater. Chem. Phys. 2002, 77, 895

(32) Li, X.; Lu, X.; Lu, Q. Appl. Surf. Sci. 2007, 253, 3690.

(33) Mate, C. M.; Toney, M. F.; Leach, K. A. IEEE Trans. Magn. 2001, 37, 1821.

(34) Tsutsumi, N.; Fujihara, A. J, Appl. Phys. 2007, 101, 033110.

(35) Sanz, A.; Nogales, A.; Ezquerra, T; Soccio, M.; Munari, A.; Lotti, N. Macromolecules 2010, 43, 671.

(36) Vogel, A.; Venugopalan, V. Chem. Rev. 2003, 103, 577.

(37) Müller-Buschbaum, P. In Applications of Synchrotron Light to Non-Crystalline Diffraction in Materials and Life Science; Ezquerra, T. A., García-Gutiérrez, M. C., Nogales, A., Gómez, M., Eds.; Springer: Berlin, 2009; p 63.

(38) Förster, S.; Timmann, A.; Konrad, M.; Schellbach, C.; Meyer, A.; Funari, S. S.; Mulvaney, P.; Knott, R. J. Phys. Chem. B 2005, 109, 1347.

(39) Renaud, G.; Lazzari, R.; Leroy, F. Surf. Sci. Rep. 2009, 64, 255.

(40) The program IsGISAXS with instructions is available on simple request from the author R. Lazzari (http://www.insp.jussieu.fr/axe2/ Oxydes/IsGISAXS/isgisaxs.htm) (accessed March 15, 2010).

(41) Hindeleh, A. M.; Hosemann, R. J. Mater. Sci. 1991, 6, 5127.

(42) Mark, J. E. Physical Properties of Polymers Handbook; Springer: Berlin, 2007. 